\title{
An Integrated Differentiation-Projection Approach for the kinematic Data Consistency of biomechanical Systems
}

\author{
F.J. Alonso, J. Cuadrado and P. Pintado
}

\begin{abstract}
Several sources of error corrupt the results obtained in the kinematic and dynamic analysis of biomechanical systems and reduce its usefulness. The main source of error is the inaccuracy of velocities and accelerations derived from experimentally measured displacements of markers placed on the skin of joints. This error is mainly due to the amplification of high-frequency low-amplitude noise introduced by the motion capture system when the raw displacement signals are differentiated. Another source of error is the skin motion artifact, that produces violations of the kinematic constraint equations of the multibody system. An integrated differentiation-projection approach to ensure the kinematic data consistency in the context of the analysis of biomechanical systems is presented. The raw data differentiation problem is solved by applying a smoothing-differentiation technique based on the Newmark integration scheme. Several benchmark kinematic signals that include computer generated data of a four-bar mechanism were processed using the differentiation-projection method to study its performance.
\end{abstract}

Keywords Biomechanics, Skin motion, Kinematic consistency, Orthogonal projection methods, Raw data problem

\section{Introduction}

The inverse dynamic analysis (IDA) of biomechanical systems uses kinematic and anthropometric data to calculate net joint reaction forces and net driver moments during a physical activity or motion.

The estimation of the skeletal motion obtained from marker-based motion capture systems is known to be affected by significant errors caused by skin motion artifact [1], i.e. the motion of the skin with respect to the underlying bone, and due

\section{F.J. Alonso}

Departamento de Ingeniería Mecánica, Energética y de los Materiales, Universidad de Extremadura, Avda. de Elvas s/n, 06071 Badajoz, Spain

e-mail: fjas@unex.es 
to the amplification of high-frequency low-amplitude noise introduced by the motion capture system when the raw displacement signals are differentiated to obtain velocities and accelerations [2].

To avoid raw data amplification during differentiation, the acquired displacement signals are smoothed prior to differentiation. The problem of filtering displacement time series to obtain noiseless velocities and accelerations with minimum loss of information has been widely studied. Digital Butterworth filters, splines, and filters based on spectral analysis among traditional smoothing methods [2, 3] and advanced smoothing techniques like discrete wavelet transform or SSA [4] have been applied and tested. Nevertheless, the smoothing procedure does not ensure the kinematic data consistency with the biomechanical model because the kinematic constraint equations are not necessarily satisfied.

In this work we propose the application of an integrated differentiation-projection approach to ensure the kinematic data consistency of biomechanical systems. The new procedure smooths and differentiates the kinematic signals in a single step using the Newmark integration scheme and then projects the positions and the obtained velocities and accelerations to the constraint manifold.

\section{The Newmark Method}

The Newmark method is a single-step integration formula. The method constitutes a special category of finite difference methods that have been widely used in solving the multi-DOF second-order differential equations that appear in structural dynamics.

The state vector of the system at a time $t_{n+1}=t_{n}+h$ is deduced from the alreadyknown state vector at time $t_{n}$, through a Taylor expansion of the displacements and velocities [5]. The following are the two basic equations proposed by Newmark [5] for determining displacements and velocities of the structure at time $t_{n+1}$ :

$$
\begin{gathered}
\dot{\mathbf{q}}_{n+1}=\dot{\mathbf{q}}_{n}+h(1-\gamma) \ddot{\mathbf{q}}_{n}+\gamma \ddot{\mathbf{q}}_{n+1} \\
\mathbf{q}_{n+1}=\mathbf{q}_{n}+h \dot{\mathbf{q}}_{n}+h^{2}\left[\left(\frac{1}{2}-\beta\right) \ddot{\mathbf{q}}_{n}+\beta \ddot{\mathbf{q}}_{n+1}\right]
\end{gathered}
$$

The constants $\gamma$ and $\beta$ are parameters associated with the quadrature scheme. The parameter $\beta$ denotes the variation of the acceleration during the incremental time steph $=t_{n+1}-t_{n}$. The choice of $\beta$ implies different schemes of interpolation for the acceleration over a time step. The value $\beta=0$ indicates a scheme equivalent to the central difference method, $\beta=1 / 4$ is a constant average acceleration method, and the value $\beta=1 / 6$ is a linear acceleration method. The parameter $\gamma$ relates to the numerical damping introduced by discretization in the time domain. For the case with $\gamma<1 / 2$, there exists some negative numerical damping while, for $\gamma>1 / 2$, 
positive numerical damping will occur [5]. The method has been demonstrated to be unconditionally stable when:

$$
\begin{gathered}
\gamma \geq \frac{1}{2} \\
\beta \geq \frac{1}{4}(0.5+\gamma)^{2}
\end{gathered}
$$

From Eqs. (1) and (2), the accelerations and velocities of the structure at time step $t_{n+1}$ can be solved as

$$
\begin{gathered}
\ddot{\mathbf{q}}_{n+1}=\frac{1}{\beta h^{2}}\left(\mathbf{q}_{n+1}-\mathbf{q}_{n}\right)-\frac{1}{\beta h} \dot{\mathbf{q}}_{n}-\left(\frac{1}{2 \beta}-1\right) \ddot{\mathbf{q}}_{n} \\
\dot{\mathbf{q}}_{n+1}=\dot{\mathbf{q}}_{n}+h(1-\gamma) \ddot{\mathbf{q}}_{n}+h \gamma \ddot{\mathbf{q}}_{n+1}
\end{gathered}
$$

\section{Kinematic Data Consistency}

The skin motion artifact affects the kinematics of the multibody system, producing violations of the kinematic constraint equations. This violation is called kinematic data inconsistency and is regarded as one of the most critical sources of error in human movement analysis [6,7]. This inconsistency produces spurious reaction forces and driver moments when the inverse dynamic analysis is performed.

A mechanical system is consistent with the acquired kinematic data when the constraint equations and their time derivatives are satisfied [6]. Silva and Ambròsio [6] applied a systematic procedure using a multibody formalism to ensure kinematic data consistency. They use the non-consistent $\mathbf{q}^{*}$ positions as an initial guess to the Newton-Raphson procedure to obtain consistent positions:

$$
\Phi_{\mathbf{q}}\left(\mathbf{q}_{i}\right) \Delta \boldsymbol{q}_{i}=-\Phi\left(\mathbf{q}_{i}\right)
$$

Where $\Delta \boldsymbol{q}_{i}=\mathbf{q}-\mathbf{q}_{i}$ is the generalized coordinates correction at iteration $i$. Consistent velocities $\dot{\mathbf{q}}$ and accelerations $\ddot{\mathbf{q}}$ are obtained by solving velocity and acceleration equations of the multibody system:

$$
\begin{aligned}
& \Phi_{\mathbf{q}}(\mathbf{q}) \dot{\mathbf{q}}=\mathbf{0} \\
& \Phi_{\mathbf{q}}(\mathbf{q}) \ddot{\mathbf{q}}=-\dot{\Phi}_{\mathbf{q}} \dot{\mathbf{q}}
\end{aligned}
$$

This procedure produces reasonable good results [6]. Nevertheless, the biomechanical model is driven by the angular histories calculated from the inconsistent input 
data, which are not the true angular histories. To overcome the calculation of the intersegmental angles from inconsistent data, this work proposes the simultaneous correction of the natural coordinates and intersegmental angles performing an orthogonal projection of the position solution to the constraint manifold, in order to obtain a new set of positions $\mathbf{q}$ that satisfies $\Phi=\mathbf{0}$. This scheme projects the subset of natural coordinates measured with the motion capture system and calculates the angular histories from the consistent positions. The projection can be obtained by the solution of the following constrained minimization problem [6]:

$$
\min _{\mathbf{q}} V=\frac{1}{2}\left(\mathbf{q}-\mathbf{q}^{*}\right)^{T} \mathbf{W}\left(\mathbf{q}-\mathbf{q}^{*}\right) \quad \text { s.t. } \Phi=\mathbf{0}
$$

Where $\mathbf{W}$ is a weighting matrix. Different weighting factors can be assigned to each natural coordinate to reflect the average degree of skin movement artifact associated to each coordinate. An identity weighting matrix has been used in this work. Using an augmented Lagrangian method to minimize the above function [8], the following iterative scheme to calculate the consistent data positions $q$ is obtained:

$$
\left[\mathbf{W}+\Phi_{\mathbf{q}}^{T} \alpha \Phi_{\mathbf{q}}\right] \Delta \boldsymbol{q}_{i+1}=-\mathbf{W D q} \mathbf{q}_{i}-\Phi_{\mathbf{q}}^{T} \alpha \Phi
$$

Where $\Delta \boldsymbol{q}_{i+1}$ and $\Delta \mathbf{q}_{i}$ are the position data corrections and the subscripts indicate the iteration number. Equation (10) can be solved iteratively until $\left\|\Delta \mathbf{q}_{i}\right\|<\varepsilon$ where $\varepsilon$ is a user specified tolerance. The value of the penalty factor $\alpha$ only affects the convergence rate. Bayo et al. [8] recommend penalty factors ranging from $10^{5}$ to $10^{7}$ to obtain a good convergence rate. The execution times to solve Eqs. (6) and (10) were similar. In order to obtain consistent velocities $\dot{\mathbf{q}}$ again we perform an orthogonal projection of the velocities $\dot{\mathbf{q}}^{*}$ calculated using the Newmark scheme to the velocity constraint manifold. This can be achieved by the solution of the linear equation:

$$
\left[\mathbf{W}+\Phi_{\mathbf{q}}^{T} \alpha \Phi_{\mathbf{q}}\right] \dot{\mathbf{q}}=\mathbf{W} \dot{\mathbf{q}}^{*}
$$

To obtain consistent accelerations, the projection of the accelerations $\ddot{\mathbf{q}}^{*}$ calculated using the Newmark scheme onto the constraint manifold can be obtained trough the solution of the following equation:

$$
\left[\mathbf{W}+\Phi_{\mathbf{q}}^{T} \alpha \Phi_{\mathbf{q}}\right] \ddot{\mathbf{q}}=\mathbf{W} \ddot{\mathbf{q}}^{*}-\Phi_{\mathbf{q}}^{T} \alpha \dot{\Phi}_{\mathbf{q}} \dot{\mathbf{q}}
$$

\section{Results}

To test the performance of the proposed procedure several benchmark raw displacement signals (observed signals) were processed using the smoothing-differentiation procedure and the projection methods described in the previous sections. The computer-generated data include the simulation of a four-bar crank-rocker mech- 
anism during two crank revolutions (Fig. 1a) The input angular velocity and the lengths of the links were fixed to $\dot{\theta}=2 \pi \mathrm{rad} / \mathrm{s}, L_{1}=2 \mathrm{~m}, L_{2}=8$ mand $L_{3}=5 \mathrm{~m}$. The time step $h=0.01 \mathrm{~s}$ and the total time of simulation is $2 \mathrm{~s}$. The original data $q=\left(x_{1}, y_{1}, x_{2}, y_{2}\right)^{T}$ were corrupted to simulate skin motion artifact and noise introduced by the motion capture system using three different patterns: Gaussian noise, Sinusoidal stationary noise and Non-stationary noise. The non-stationary noise is simulated by adding lumped point masses connected to the system by viscoelastic unions. Figure 1a, 1b, 1c, 1d, 1e and 1f show the original data and the corrupted data.

a)

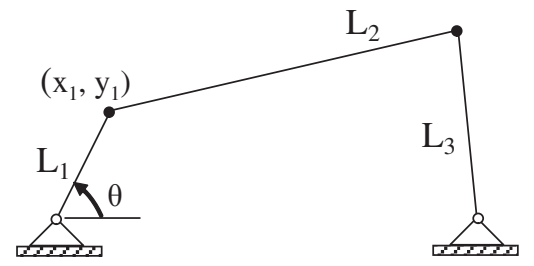

c)

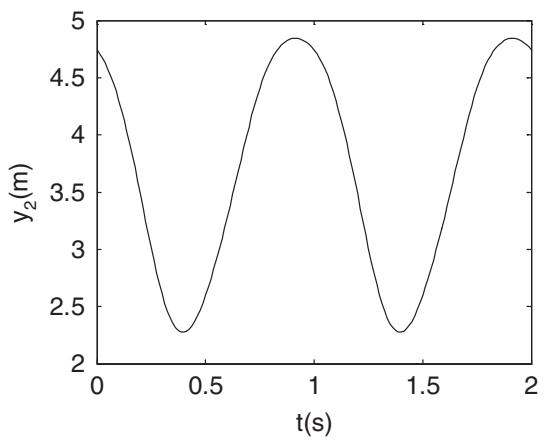

e)

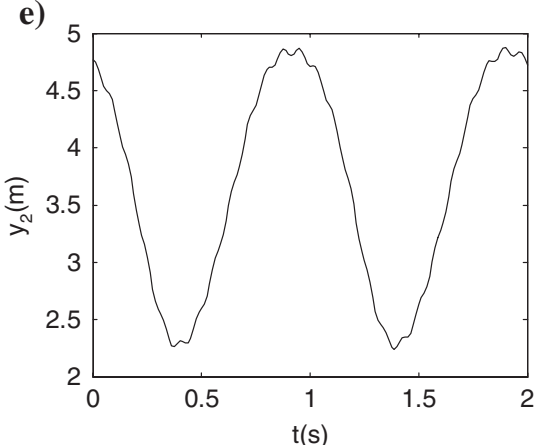

b)

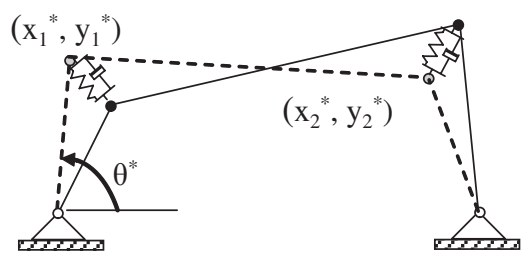

d)

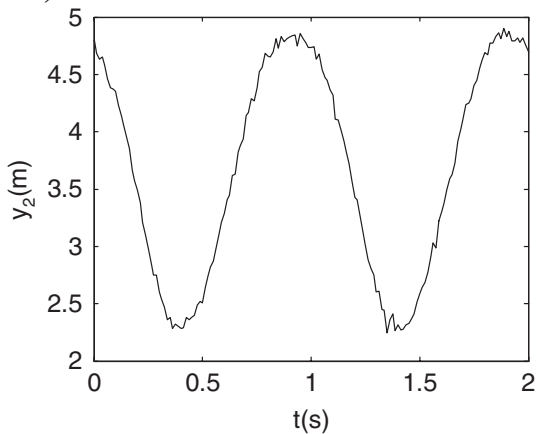

f)

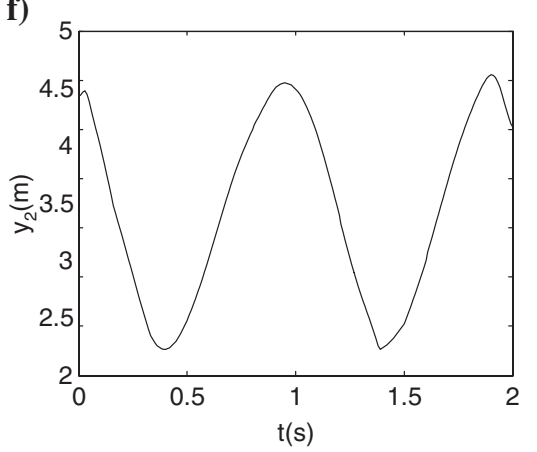

Fig. 1 The four-bar example: (a) original coordinates; (b) model for the simulation of nonstationary noise; (c) the original $y_{2} ;$ (d) the corrupted $y_{2}^{*}$ using Gaussian noise; (e) the corrupted $y_{2}^{*}$ using sinusoidal stationary noise; (f) the corrupted $y_{2}^{*}$ using non-stationary noise 
To perform the double differentiation using the Newmark scheme, several $\gamma$ and $\beta$ parameters in the stability region Eqs. (3) and (4) were chosen, and it was found that for $\gamma=3.5$ and $\beta=10 / 4(0.5+\gamma)^{2}=40$ good results were obtained. Moreover, the results obtained by using the projection methods are compared with those obtained following the procedure proposed by Silva and Ambròsio [6]. In order to compare the results obtained by different procedures we use the normalized root mean square (rms) of the residuals produced corresponding to the original coordinate $q_{i}$ :

$$
\begin{aligned}
& \eta_{i}=\sqrt{\sum_{i=1}^{N}\left[q_{i}-q_{i}^{*}\right]^{2} / \sum_{i=1}^{N} q_{i}^{2}} \\
& \dot{\eta}_{i}=\sqrt{\sum_{i=1}^{N}\left[\dot{q}_{i}-\dot{q}_{i}^{*}\right]^{2} / \sum_{i=1}^{N} \dot{q}_{i}^{2}} \\
& \ddot{\eta}_{i}=\sqrt{\sum_{i=1}^{N}\left[\ddot{q}_{i}-\ddot{q}_{i}^{*}\right]^{2} / \sum_{i=1}^{N} \ddot{q}_{i}^{2}}
\end{aligned}
$$

Figure 2a shows the results obtained for the Gaussian noise (amplitude $p=5 L_{1} / 100$ ) using the following procedures:

- Ensuring the kinematic consistency using the method proposed by Silva and Ambròsio [6] (KC).

- Using the projection methods for $q=\left(x_{1}, y_{1}, x_{2}, y_{2}\right)^{T}$ and its higher derivatives $(\mathrm{KCP})$.

Figure 2a shows that the proposed projection process improves the results obtained by conventionally imposing kinematic consistency using Eqs. (6) and (8). Moreover, the application of the Newmark scheme to calculate the higher derivatives prior to projection dramatically improves the results, as shown in Fig. 2b. This fact illustrates the importance of the raw displacement smoothing and differentiation method in this problem. Table 1 summarizes results for the sinusoidal stationary noise and non-stationary noise using KC (regular face) and KCP methods (boldface) and using the Newmark integration scheme with $\gamma=3.5$ and $\beta=10 / 4(0.5+\gamma)^{2}=40$ to calculate the higher derivatives.

\section{Conclusion}

A systematic multibody procedure based on differentiation-smoothing using the Newmark scheme and orthogonal projection of the position, velocity and acceleration to the constraint manifold to ensure the kinematic data consistency in the 
(a)

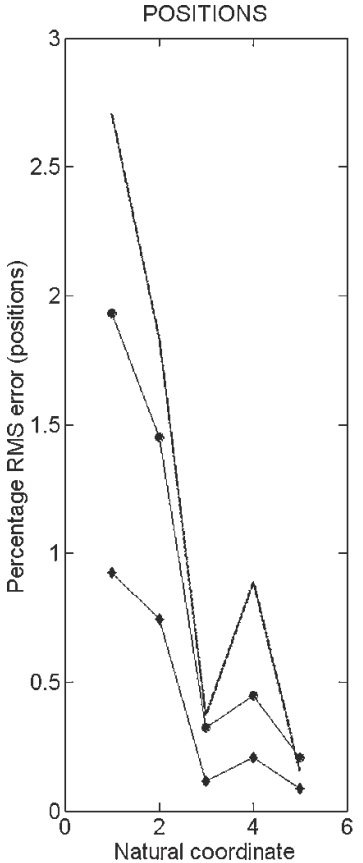

(b)

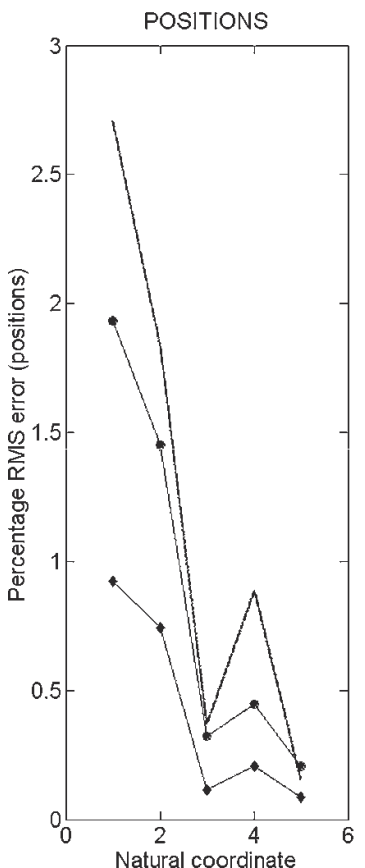

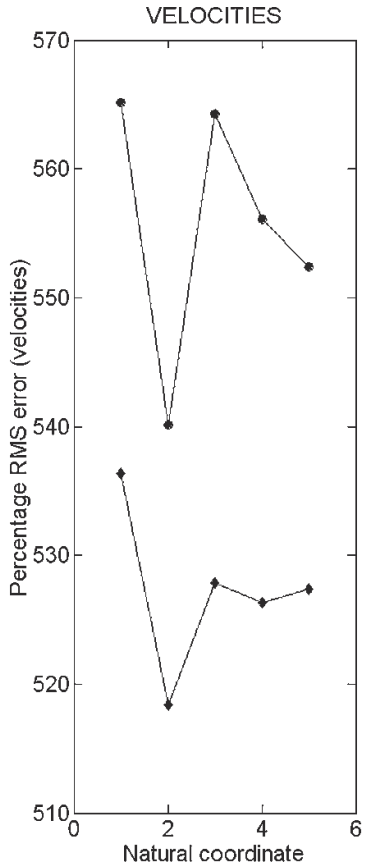
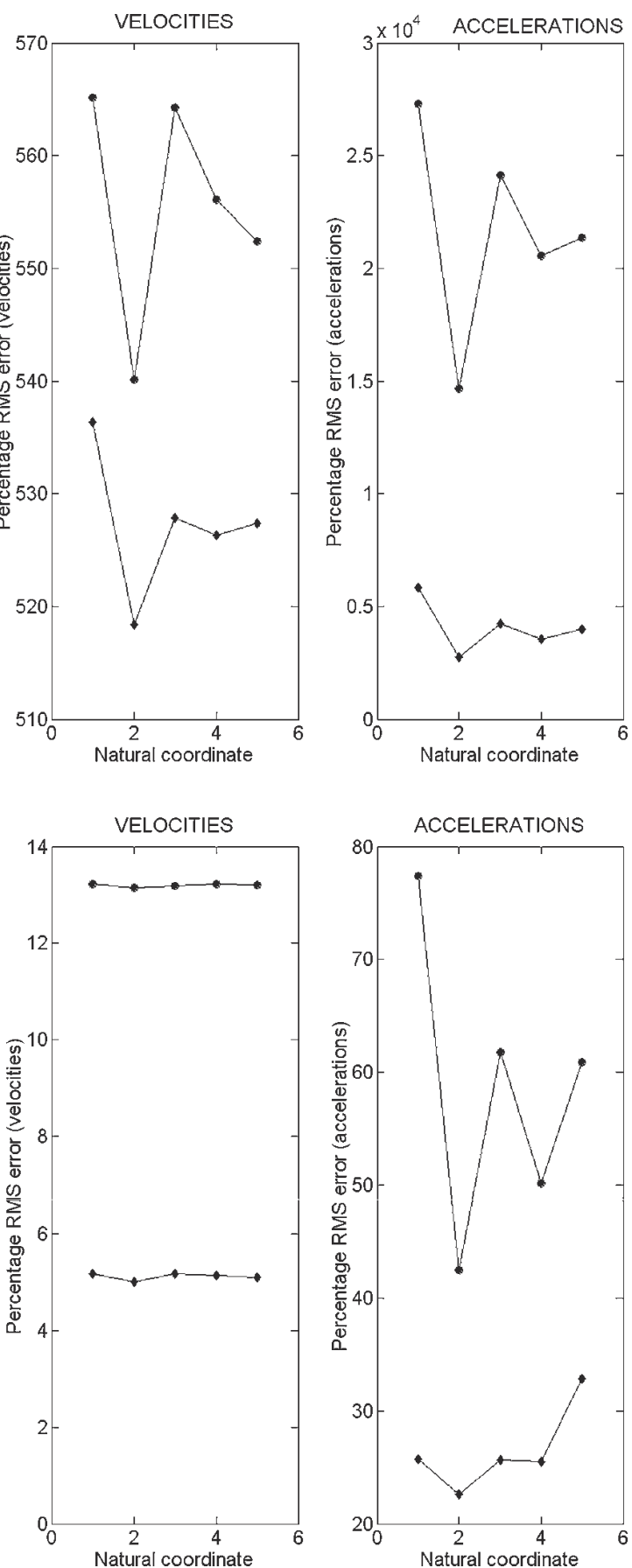

Fig. 2 Residuals: (a) No smoothing technique applied; (b) Newmark-smoothed signals. (•) KC, ( $\mathrm{KCP}$. The dashed line in the positions plots correspond to the corrupted position data 
Table 1 Summary of the results for the sinusoidal and non-stationary cases. KC in regular face, $\mathrm{KCP}$ in bold face

\begin{tabular}{clll}
\hline Coordinate & $\mathbf{q}$ & $\dot{\mathbf{q}}$ & $\ddot{\mathbf{q}}$ \\
\hline Sinusoidal & $\mathrm{KC}(\mathbf{K C P})$ & $\mathrm{KC}(\mathbf{K C P})$ & $\mathrm{KC}(\mathbf{K C P})$ \\
\hline & $7.41(\mathbf{3 . 5 9})$ & $11.23(\mathbf{7 . 6 6})$ & $70.54(\mathbf{3 0 . 1 9})$ \\
1 & $5.53(\mathbf{3 . 2 6})$ & $10.72(\mathbf{6 . 2 3})$ & $59.12(\mathbf{2 9 . 0 8})$ \\
2 & $1.15(\mathbf{0 . 6 8})$ & $9.01(\mathbf{7 . 1 4})$ & $60.94(\mathbf{2 7 . 2 9})$ \\
3 & $1.20(\mathbf{0 . 7 4})$ & $11.84(\mathbf{9 . 5 8})$ & $54.19(\mathbf{1 7 . 2 1})$ \\
4 & $0.50(\mathbf{0 . 3 2})$ & $10.45(\mathbf{6 . 9 5})$ & $47.83(\mathbf{2 3 . 1 6})$ \\
5 & & & \\
Non-Stationary & $\mathrm{KC}(\mathbf{K C P})$ & $\mathrm{KC}(\mathbf{K C P})$ & $\mathrm{KC}(\mathbf{K C P})$ \\
\hline & & & \\
1 & $1.46(\mathbf{1 . 3 5})$ & $40.17(\mathbf{3 8 . 1 2})$ & $52.14(\mathbf{2 9 . 7 5})$ \\
2 & $1.33(\mathbf{1 . 3 2})$ & $39.15(\mathbf{3 1 . 8 2})$ & $45.85(\mathbf{2 3 . 5 7})$ \\
3 & $0.23(\mathbf{0 . 2 2})$ & $41.52(\mathbf{2 9 . 1 4})$ & $46.05(\mathbf{2 9 . 7 3})$ \\
4 & $0.31(\mathbf{0 . 2 7})$ & $46.73(\mathbf{3 4 . 5 9})$ & $52.66(\mathbf{2 2 . 1 8})$ \\
5 & $0.17(\mathbf{0 . 1 6})$ & $42.68(\mathbf{3 5 . 2 9})$ & $33.75(\mathbf{2 7 . 2 2})$ \\
\hline
\end{tabular}

context of the analysis of biomechanical systems is presented. The results show the superiority of these methods over other multibody procedures. Future works will need to focus on the possibilities of producing an automatic algorithm, and on the embedding the algorithm in commercial biomechanical analysis packages. Namely, techniques will be devised to automatically choose the $\gamma$ and $\beta$ parameters for smoothing and differentiation of the given signal. The next step is to ensure the kinematic data consistency using a detailed body model that accounts for the flexibility of the real biomechanical system bodies and joints.

\section{References}

1. Hatze, H., The fundamental problem of myoskeletal inverse dynamics and its implications, Journal of Biomechanics, Vol. 35, 2002, pp. 109-115.

2. Vaughan, C. L., Smoothing and differentiation of displacement-time data: an application of splines and digital filtering, International Journal of Bio-Medical Computing, Vol. 13, 1982, pp. $375-386$.

3. Dowling, J., A modelling strategy for the smoothing of biomechanical data, In: B. Johnsson, (Ed.), Biomechanics, Vol. XB. Human Kinetics, Champaign, IL, pp. 1163-1167, 1985.

4. Alonso, F. J., Del Castillo, J. M., Pintado, P., Application of singular spectrum analysis to the smoothing of raw kinematic signals, Journal of Biomechanics, Vol. 38, 2005, pp. 1085-1092.

5. Newmark, N. M., A method of computation for structural dynamics, Journal of Engineering Mechanics Division, Proceedings of ASCE, Vol. 85 (EM3), 1959, pp. 67-94.

6. Silva, M. P. T., Ambròsio, J. A. C., Kinematic data consistency in the inverse dynamic analysis of biomechanical systems, Multibody System Dynamics, Vol. 8, 2002, pp. 219-239.

7. Alonso, F. J., Del Castillo, J. M., Pintado, P., Motion data processing and wobbling mass modelling in the inverse dynamics of skeletal models, Mechanism and Machine Theory, Vol. 42, 2007, pp. 1153-1169.

8. Bayo, E., Ledesma, R., Augmented lagrangian and mass-orthogonal projection methods for constrained multibody dynamics, Nonlinear Dynamics, Vol. 9, 1996, pp. 113-130. 